\title{
Bidirectional modulation of insulin action by reactive oxygen species in 3T3-L1 adipocytes
}

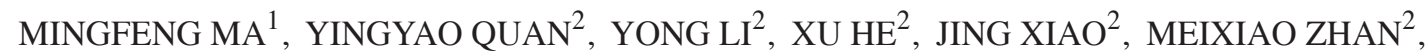 \\ WEI ZHAO ${ }^{2}$, YONGJIE XIN ${ }^{2}$, LIGONG LU ${ }^{2}$ and LIANGPING LUO ${ }^{3}$ \\ ${ }^{1}$ Department of Cardiology, Zhuhai Hospital Affiliated with Jinan University (Zhuhai People's Hospital); \\ ${ }^{2}$ Center of Intervention Radiology, Zhuhai Precision Medicine Center, Zhuhai People's Hospital, Zhuhai, \\ Guangdong 519000; ${ }^{3}$ Department of Medical Imaging Center, The First Affiliated Hospital of Jinan University, \\ Guangzhou, Guangdong 510630, P.R. China
}

Received January 1, 2018; Accepted May 4, 2018

DOI: $10.3892 / \mathrm{mmr} .2018 .9016$

\begin{abstract}
Reactive oxygen species (ROS) serve an important role in glucose-lipid metabolic regulation. In the present study, the results demonstrated that there was bidirectional regulation of insulin action in 3T3-L1 adipocytes treated with ROS. Transient and acute ROS exposure improved insulin-induced metabolic effects in 3T3-L1 adipocytes. Hydrogen peroxide $\left(\mathrm{H}_{2} \mathrm{O}_{2}\right)$, as a stable and diffusible ROS, diffused into adipocytes and altered intracellular redox homeostasis, resulting in oxidation and inactivation of phosphatase and tensin homologue deleted on chromosome 10 (PTEN). Inactivation of PTEN enhanced the activation of insulin-induced protein kinase B (AKT), leading to increased glucose transporter 4 (GLUT4) redistribution and glucose uptake in 3T3-L1 adipocytes.
\end{abstract}

Correspondence to: Professor Ligong Lu, Center of Intervention Radiology, Zhuhai Precision Medicine Center, Zhuhai People's Hospital, 79 Kangning Road, Zhuhai, Guangdong 519000 , P.R. China

E-mail:1lg0902@sina.com

Professor Liangping Luo, Department of Medical Imaging Center, The First Affiliated Hospital of Jinan University, 613 Huangpu West Road, Guangzhou, Guangdong 510630, P.R. China

E-mail: liangpingluo@126.com

Abbreviations: ROS, reactive oxygen species; PTEN, phosphatase and tensin homologue deleted on chromosome 10; GLUT4, glucose transporter 4; ER, endoplasmic reticulum; PTPs, protein tyrosine phosphatases; PI3K, phosphoinositide-3 kinase; IR, insulin receptor; IRS, insulin receptor substrate; NAC, N-acetylcysteine; NEM, $\mathrm{N}$-ethylmaleimide; FBS, fetal bovine serum; PTP1b, protein-tyrosine phosphatase-1b; MAPKs, mitogen-activated protein kinases; JNK, c-Jun N-terminal kinase; ASK1, apoptosis signal-regulating kinase 1; TRX, oxidizing thioredoxin; MFI, mean fluorescence intensity; MTR, MitoTraker Deeper Red; PBS, phosphate-buffered saline; NADPH, nicotinamide adenine dinucleotide phosphate; $\mathrm{H}_{2} \mathrm{O}_{2}$, hydrogen peroxide; IKK $\beta$, nuclear factor $\kappa$-B kinase subunit $\beta$

Key words: reactive oxygen species, insulin sensitivity, 3T3-L1 adipocytes, glucose transporter 4 , glucose uptake
However, chronic ROS treatment induced insulin resistance in 3T3-L1 adipocytes. It was also revealed that insulin-induced AKT activation, GLUT4 translocation to cell membrane and glucose uptake were significantly inhibited in chronic ROS-treated 3T3-L1 adipocytes. Taken together, the present study provided further demonstration that transient ROS treatment improved insulin sensitivity; however, chronic ROS exposure induced insulin resistance in 3T3-L1 adipocytes.

\section{Introduction}

Reactive oxygen species (ROS) have traditionally been viewed as an undesirable metabolic byproduct, which are principally generated by the mitochondrial respiratory chain and plasma membrane enzyme system (1). In obesity and type 2 diabetes mellitus, a high caloric diet causes a surplus of reducing equivalents, increasing electron transport in the mitochondrial respiratory chain and ROS generation (2-4). Other studies show that the endoplasmic reticulum (ER) is a potentially important source of $\operatorname{ROS}(2,5,6)$. The ER is an important site of protein folding and disulfide bond formation in eukaryotic organisms (7). The oxidation of cysteine residues and the release of electrons are involved in the formation of disulfide bonds, which results in the production of superoxide anions $\left(\mathrm{O}_{2}^{\circ}\right)$ and other $\operatorname{ROS}(2,5)$. However, nutrient excess might overload the protein-folding ability of the ER and cause chronic ROS generation, resulting in insulin resistance, obesity and type 2 diabetes mellitus (2). Therefore, chronic ROS generation and oxidative stress can suppress the insulin response and contribute to the development of insulin resistance.

Although intercellular chronic oxidative stress induces insulin resistance in obesity and type 2 diabetes mellitus, ROS have an important role in insulin-regulated glucose and lipid metabolism $(1,8,9)$.Previous studies reported that insulin activates nicotinamide adenine dinucleotide phosphate (NADPH) oxidase in the plasma membrane, leading to hydrogen peroxide $\left(\mathrm{H}_{2} \mathrm{O}_{2}\right)$ generation $(10,11)$. The protein tyrosine phosphatase (PTP) family is oxidized and inactivated by ROS, which are dephosphorylases and catalyze the dephosphorylation of tyrosyl-phosphorylated proteins (12). Phosphatase and tensin homologue deleted on chromosome 10 (PTEN), one member of the PTP family, 
can dephosphorylate phosphatidylinositol-3-phosphate (PIP), phosphatidylinositol-3,4-bisphosphate $\left(\mathrm{PIP}_{2}\right)$ and phosphatidylinositol-3,4,5-triphosphate $\left(\mathrm{PIP}_{3}\right)$ in vitro, while it is likely that $\mathrm{PIP}_{3}$ is the most important substrate in vivo $(13,14)$. Activation of insulin-induced phosphoinositide-3 kinase (PI3-K) catalyzes $\mathrm{PIP}_{3}$ generation to induce the phosphorylation and activation of protein kinase B (AKT) $(15,16)$. Thus, PTEN and PI3-K have opposing effects on $\mathrm{PIP}_{3}$ content (17), and inactivation of PTEN enhances insulin-stimulated metabolic effects (18).

In insulin-signaling processes, insulin-induced insulin receptor (IR) phosphorylates insulin receptor substrate (IRS) proteins, which further phosphorylate the PI3-K regulatory subunit and activate the PI3-K catalytic subunit. PI3-K activation converts $\mathrm{PIP}_{2}$ to $\mathrm{PIP}_{3}$, which results in the activation of AKT (19). Subsequently, PI3-K/AKT signaling regulates glucose uptake, glycogen synthesis and lipid synthesis (15). In adipose cells and tissues, insulin-activated AKT signaling pathways promotes glucose transporter 4 (GLUT4) translocation to the cell membrane, increasing extracellular glucose uptake (20). However, GLUT4 defects or reduced expression in adipose cells and tissues can cause insulin resistance and metabolic syndrome in mouse models and patients $(21,22)$. Thus, regulation of glucose uptake in fatty tissues has an important role in the development of whole-body insulin resistance and type 2 diabetes mellitus. In the present study, we find that transient $\mathrm{H}_{2} \mathrm{O}_{2}$ stimulation increases insulin sensitivity in 3T3-L1 adipocytes. $\mathrm{H}_{2} \mathrm{O}_{2}$ treatment oxidizes and inhibits PTEN, enhancing insulin-induced AKT phosphorylation, GLUT4 translocation and glucose uptake in 3T3-L1 adipocytes. However, chronic ROS exposure attenuates the insulin-stimulated AKT signaling pathway and causes insulin resistance in 3T3-L1 adipocytes. Our data indicate different effects of ROS on insulin-regulated signaling pathways in 3T3-L1 adipocytes.

\section{Materials and methods}

Materials. Dulbecco's modified Eagle's medium/Ham's nutrient mixture F12 (DMEM/F12) were purchased from Gibco Co.Ltd, Grand Island, NY, USA. Methylisobutylxanthine, dexamethasone and insulin were purchased from Tocris Bioscience (Bristol, UK). Oil Red O, [2-(N-(7-nitrobenz-2-oxa-1,3-diazol-4-yl) amino)-2-deoxyglucose] (2-NBDG), $\mathrm{N}$-acetylcysteine (NAC), N-ethylmaleimide (NEM) and glucose oxidase were purchased from Sigma-Aldrich (St. Louis, MO, USA). $\mathrm{H}_{2} \mathrm{O}_{2}$ was purchased from Guangzhou Chemical Reagent Factory, (Guangzhou, China). $\mathrm{H}_{2}$ DCFDA and MitoTracker Red were purchased from Thermo Fisher Scientific, Inc. (Pittsburgh, PA, USA). Anti-PTEN (cat. no. 5384), anti-phospho-Thr308/309-AKT [AKT (T308/309P); cat. no. 13038)], anti-phospho-Ser473/474-AKT [AKT (S473/474P); cat. no. 9271)], anti-total-AKT (cat. no. 9272) and anti-GLUT4 (cat. no. 2213) were procured from Cell Signaling Technology, Inc. (Danvers, MA, USA). Anti- $\beta$-actin (sc-47778) was purchased from Santa Cruz Biotechnology (Santa Cruz, CA, USA). Goat Anti-Mouse IgG H\&L (Alexa Fluor 680; ab175775), Goat Anti-Rabbit IgG H\&L (Alexa Fluor 790; ab175781), Goat Anti-Mouse IgG H\&L (Alexa Fluor 555; ab150114) and Goat Anti-Rabbit IgG H\&L (Alexa Fluor 488; ab150077) were purchased from Abcam (Cambridge, MA, USA).
Cell culture and differentiation. The 3T3-L1 preadipocytes were obtained from the Chinese Academy of Science Cell Library (Shanghai, China). 3T3-L1 preadipocytes were cultured and differentiated into 3T3-L1 adipocytes as described previously (23). The preadipocytes were grown and maintained in DMEM/F12 containing 10\% fetal bovine serum (FBS), $100 \mathrm{Us} / \mathrm{ml}$ penicillin and $100 \mu \mathrm{l} / \mathrm{ml}$ streptomycin in a $5 \% \mathrm{CO}_{2}, 37^{\circ} \mathrm{C}$ constant temperature/humidity incubator. When the cells had reached confluence, the cells were transferred to differential medium, DMEM/F12 containing $10 \%$ FBS, $0.5 \mathrm{mM}$ methylisobutylxanthine, $0.25 \mu \mathrm{M}$ dexamethasone and $1 \mu \mathrm{g} / \mathrm{ml}$ insulin, for 2 days, and then were incubated in DMEM/F12 containing 10\% FBS and insulin $(1 \mu \mathrm{g} / \mathrm{ml})$ for 2 days. Subsequently, the cells were cultured in 10\% FBS-DMEM/F12 medium. The 3T3-L1 adipocytes were experimented upon 8 days after differentiation.

Oil Red $O$ staining. Oil Red O staining was performed as described previously (24). Briefly, the cells were washed with phosphate-buffered saline (PBS), and then fixed in 4\% paraformaldehyde for $30 \mathrm{~min}$. Subsequently, the cells were incubated with Oil Red O working solution for $10 \mathrm{~min}$ at room temperature, and rinsed under running tap water for 20 min. Finally, cells were sealed with nail polish and checked using a light microscope.

ROS treatment. For transient ROS treatment, 3T3-L1 adipocytes were incubated with serum-free medium supplemented with $100 \mu \mathrm{M} \mathrm{H}_{2} \mathrm{O}_{2}$ for $15 \mathrm{~min}$. Then, the cells were used for further experiments. For chronic ROS treatment, the cells were exposed to fresh medium with $100 \mathrm{mU} / \mathrm{ml}$ glucose oxidase. Glucose oxidase catalyzed the conversion of glucose to glucuronic acid and $\mathrm{H}_{2} \mathrm{O}_{2}$, which generated about $40 \mu \mathrm{M} \mathrm{H}_{2} \mathrm{O}_{2}$ for $24 \mathrm{~h}$ (25). Then, the cells were used for further experiments.

Detection of intercellular ROS. 3T3-L1 adipocytes were treated with $100 \mu \mathrm{M} \mathrm{H}_{2} \mathrm{O}_{2}$ or $10 \mathrm{nM}$ insulin for $15 \mathrm{~min}$ and washed with PBS. Pretreatment with $200 \mu \mathrm{M}$ NAC was performed for $30 \mathrm{~min}$ before $\mathrm{H}_{2} \mathrm{O}_{2}$ or insulin. Subsequently, the cells were incubated with $20 \mu \mathrm{M} \mathrm{H}_{2} \mathrm{DCFDA}$ for $15 \mathrm{~min}$. Then, the cells were detected using the LSM510 confocal microscope (Zeiss, Jena, Germany), or were harvested and detected using flow cytometry sorting (FACS; BD Biosciences, Franklin Lakes, NJ, USA).

Glucose uptake and consumption assay. Glucose uptake was performed as described previously (26). In brief, 3T3-L1 adipocytes were starved in free-serum medium for $12 \mathrm{~h}$, then treated with or without $\mathrm{H}_{2} \mathrm{O}_{2}$ or/and insulin for $30 \mathrm{~min}$. Glucose uptake was measured using 2-NBDG. The cells were incubated with $10 \mathrm{mM}$ 2-NBDG in PBS for $15 \mathrm{~min}$ at $37^{\circ} \mathrm{C}$ and extracted using a lysis buffer. 2-NBDG content was detected using a microplate fluorimeter (Infinite M200; Tecan, Hillsborough, NC, USA). Glucose consumption in the medium was performed using the Glucose Oxidase Method (GOM; Applygen Technologies Inc., Beijing, China). The cells were cultured in fresh serum-free medium for $3 \mathrm{~h}$ after the different treatments. The glucose content in the medium was determined using GOM.

Immunofluorescence. 3T3L-L1 adipocytes were subjected to different stimulations, then fixed with $4 \%$ paraformaldehyde 

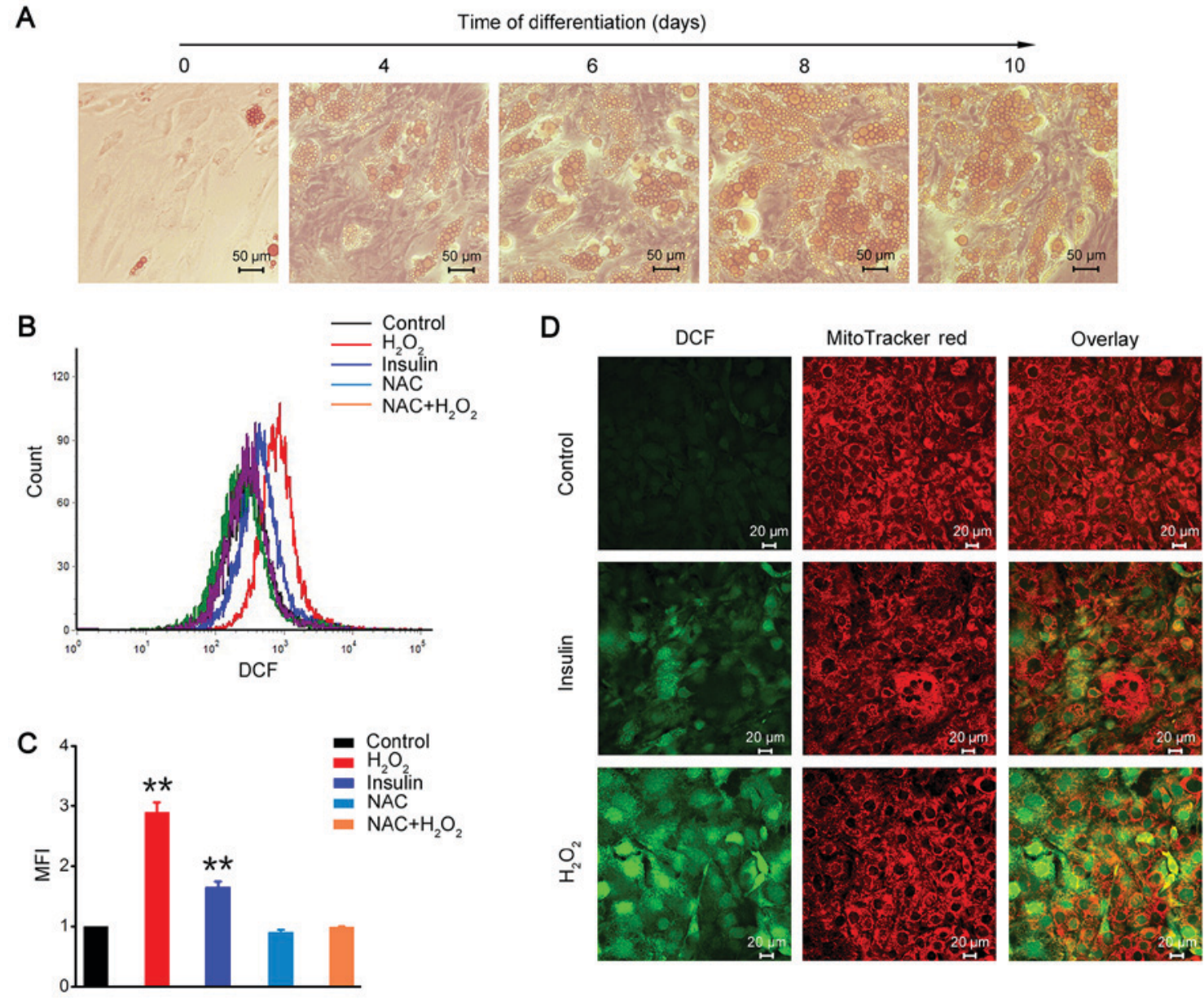

Figure $1 . \mathrm{H}_{2} \mathrm{O}_{2}$ disrupts intracellular redox balance in the same manner as insulin stimulation. (A) Representative Oil Red O staining of 3T3-L1 cells was examined using a light microscope on the indicated differentiation days (scale bar, $50 \mu \mathrm{m}$ ). (B) Flow cytometric analysis for ROS level in 3T3-L1 adipocytes in the different treatments. (C) Quantitative analysis of DCF MFI as described for panel A. Data are presented as the mean \pm standard error mean from three independent experiments. ${ }^{* *} \mathrm{P}<0.01$ vs. the control group. (D) Representative fluorescence images of intracellular ROS were obtained using confocal microscopy. ROS were stained with DCF (green), and mitochondrion were stained with MitoTraker Deeper Red (100 nM for $30 \mathrm{~min}$; red). Scale bars, $20 \mu \mathrm{m}$. $\mathrm{H}_{2} \mathrm{O}_{2}$, hydrogen peroxide; ROS, reactive oxygen species; MFI, mean fluorescence intensity; DCF, 2',7'-dichlorodihydrofluorescein; NAC, N-acetylcysteine.

for $30 \mathrm{~min}$, permeabilized with methanol for $15 \mathrm{~min}$, and incubated with anti-AKT (S473/4P) (1:300) and anti- $\beta$-actin (1:300) at $4^{\circ} \mathrm{C}$ overnight. Then, the cells were incubated with Goat Anti-Mouse IgG H\&L (Alexa Fluor 555; 1:300) for $\beta$-actin and Goat Anti-Rabbit IgG H\&L (Alexa Fluor 488; 1:300) for AKT (S473/4P) for $3 \mathrm{~h}$ at room temperature. The cells were detected using confocal microscopy.

Immunoblot analysis. Immunoblot analysis was performed as described previously (27). In brief, 3T3-L1 adipocytes were lysed using RIPA buffer (50 mM Tris-HCl (pH 8.0), $150 \mathrm{mM}$ $\mathrm{NaCl}, 0.1 \%$ SDS, $1 \%$ NP-40, 5 mM EDTA, and $0.5 \%$ sodium deoxycholate) containing $1 \mathrm{mM}$ PMSF. For the PTEN examination, cells were lysed with RIPA buffer containing $40 \mathrm{mM}$ NEM and $1 \mathrm{mM}$ PMSF. The total cell lysates were loaded onto SDS-PAGE gels and transferred to a PVDF membrane (Millipore, Billerica, MA, USA), which was incubated in the primary antibodies and the secondary antibodies. The results were detected with an ODYSSEY Infrared Imaging System (LI-COR Biosciences, Lincoln, NE, USA).

Cytoplasmandmembrane fractions. Cytoplasm and membrane fractions were obtained as described previously (28). Briefly,
3T3-L1 adipocytes were homogenized in buffer $1(10 \mathrm{mM}$ Tris- $\mathrm{HCl}, 2 \mathrm{mM} \mathrm{MgCl}_{2}, 0.2 \mathrm{M}$ sucrose, $0.5 \mathrm{mM}$ EDTA, $\mathrm{pH} 7.5$ ) and centrifuged at $1,000 \mathrm{~g}$ for $10 \mathrm{~min}$. The supernatant was centrifuged at $100,000 \mathrm{~g}$ for $40 \mathrm{~min}$ to obtain the cytoplasm fraction. The pellet was homogenized in buffer $2(6 \mathrm{mM}$ Tris-HCl, 1 mM EDTA, pH 8.0), centrifuged at 100,000 g $40 \mathrm{~min}$, and then resuspended in buffer $3(20 \mathrm{mM}$ Tris- $\mathrm{HCl}$, $1 \mathrm{mM}$ EDTA, pH 7.5) and centrifuged at 100,000 $\mathrm{g}$ for $40 \mathrm{~min}$ to obtain the membrane fraction.

Statistical analysis. All experiments were performed three times independently. Results were presented as means \pm standard error mean. Statistical analysis was performed using SPSS 13.0 software (SPSS, Inc., Chicago IL, USA) and significant differences were determined by Student's t-test or one-way analysis of variance with Tukey's post hoc test. $\mathrm{P}<0.05$ was considered to indicate a statistically significant difference.

\section{Results}

$\mathrm{H}_{2} \mathrm{O}_{2}$ disrupts the intracellular redox balance in a manner similar to insulin stimulation. 3T3-L1 preadipocytes are derived from mouse embryos and are a fibroblast cell type. The 
A

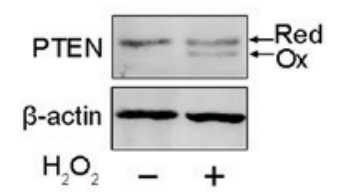

B

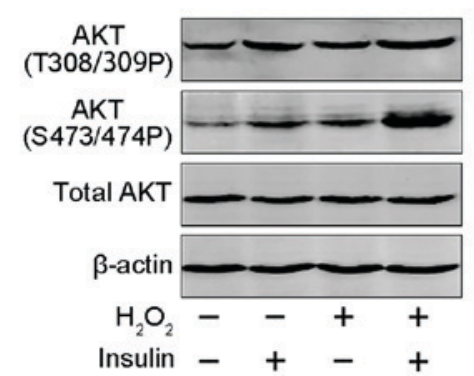

D
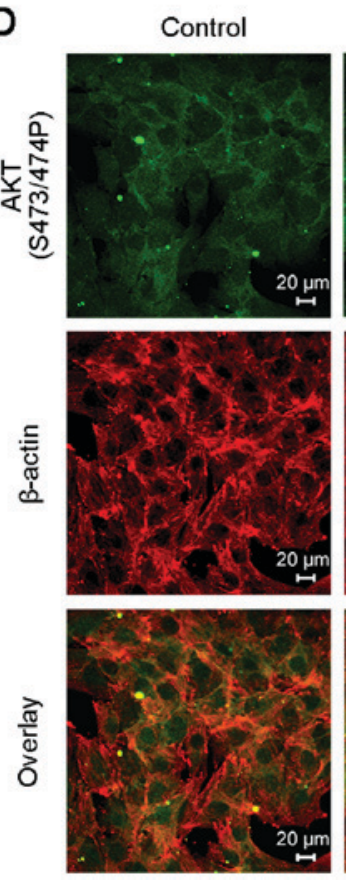
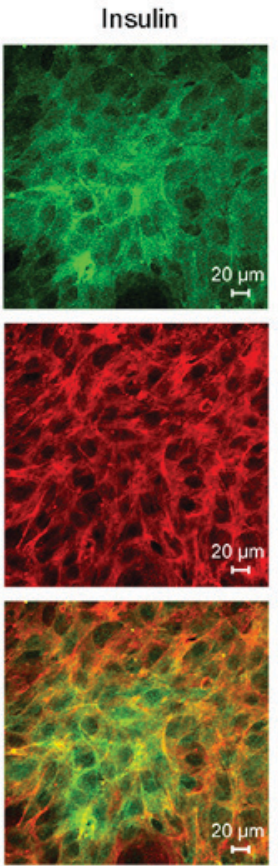

C
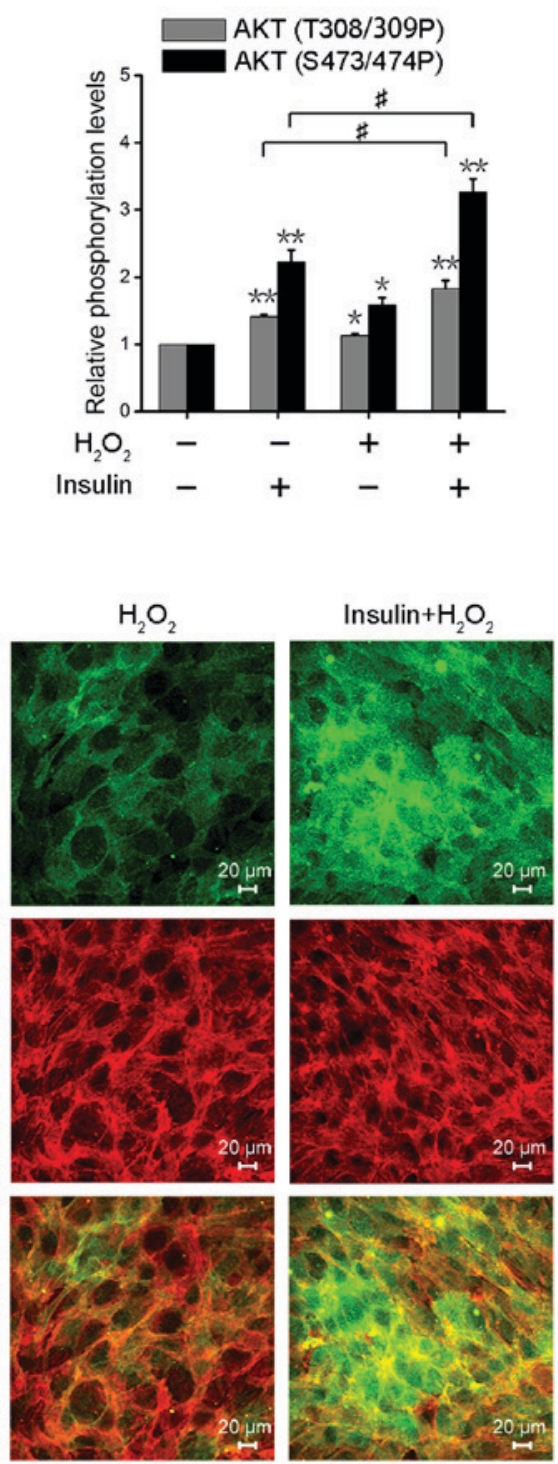

Figure 2. $\mathrm{H}_{2} \mathrm{O}_{2}$ treatment enhances insulin sensitivity in 3T3-L1 adipocytes. (A) Immunoblot analysis of PTEN oxidation in 3T3-L1 adipocytes stimulated with $\mathrm{H}_{2} \mathrm{O}_{2}$ for 5 min. (B) Immunoblot analysis of AKT phosphorylation in 3T3-L1 adipocytes under insulin and/or $\mathrm{H}_{2} \mathrm{O}_{2}$ treatments for 30 min. (C) Quantitative analysis of AKT phosphorylation protein level as presented in panel B. Data are presented as the mean \pm standard error mean from three independent experiments. ${ }^{*} \mathrm{P}<0.05$ and ${ }^{* *} \mathrm{P}<0.01$ vs. the control group; ${ }^{*} \mathrm{P}<0.05$, as indicated. (D) Representative immunofluorescence images of AKT phosphorylation in $3 \mathrm{~T} 3-\mathrm{L} 1$ adipocytes under the different treatments. Phosphorylation of AKT was stained with Anti-Rabbit IgG (green) and $\beta$-actin was stained with Anti-Mouse IgG (red). Scale bars, $20 \mu \mathrm{m} . \mathrm{H}_{2} \mathrm{O}_{2}$, hydrogen peroxide; PTEN, phosphatase and tensin homologue deleted on chromosome 10; AKT, protein kinase B; IgG, immunoglobulin G.

cells are differentiated into 3T3-L1 adipocytes, which exhibit characteristics of adipose cells, including the expression of lipid metabolic genes and the production of lipid droplets (29). Thus, this cell line is often used in lipid metabolic studies on adipose tissue. As shown in Fig. 1A, 3T3-L1 preadipocytes appeared to have a fusiform and flattened fibroblastic morphology, and converted into a 'rounded-up' morphology after differentiation. The number and size of the lipid droplets visualized by Oil Red O staining were gradually increased in a differentiating time-dependent manner (Fig. 1A). The 3T3-L1 adipocytes of 8-day differentiation were used in the subsequent experiments.

The oxidation sensitive probe $\mathrm{H}_{2} \mathrm{DCFDA}$ is often used to examine intracellular ROS levels, and is cleaved by nonspecific esterases to transform into the dichlorodihydrofluorescein derivative $\mathrm{H}_{2}$ DCF. $\mathrm{H}_{2} \mathrm{DCF}$ is oxidized and converts to fluorescent adduct DCF, and is trapped inside the cell $(30,31)$. The results of the FACS analysis showed that insulin stimulation increased ROS generation in 3T3-L1 adipocytes, and $\mathrm{H}_{2} \mathrm{O}_{2}$ could disrupt the intracellular redox balance and increase intracellular ROS level (Fig. 1B and C). However, the ROS scavenger NAC inhibited $\mathrm{H}_{2} \mathrm{O}_{2}$-induced intracellular ROS accumulation in 3T3-L1 adipocytes (Fig. 1B and C). Furthermore, we detected DCF fluorescence using confocal microscope. The results showed that fluorescence intensity was significantly increased after either insulin stimulation or $\mathrm{H}_{2} \mathrm{O}_{2}$ treatment in 3T3-L1 adipocytes (Fig. 1D). These results demonstrated that $\mathrm{H}_{2} \mathrm{O}_{2}$, similar to insulin treatment, could increase intracellular ROS generation and change the redox balance in 3T3-L1 adipocytes. 
A

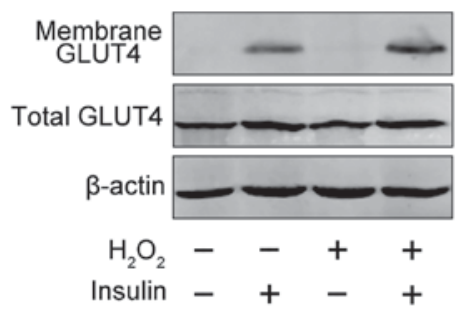

C

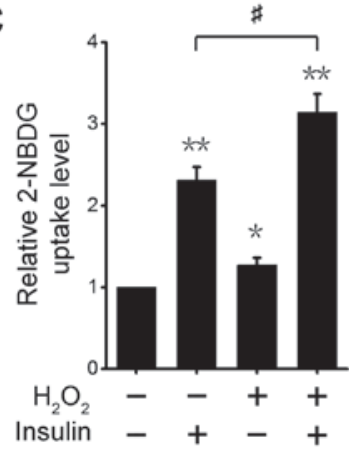

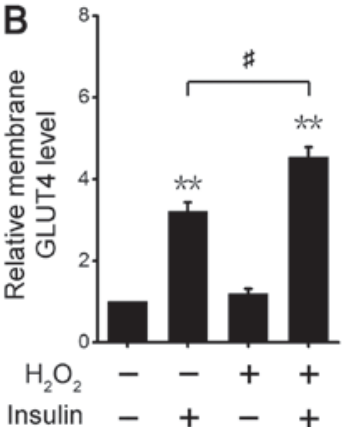

D

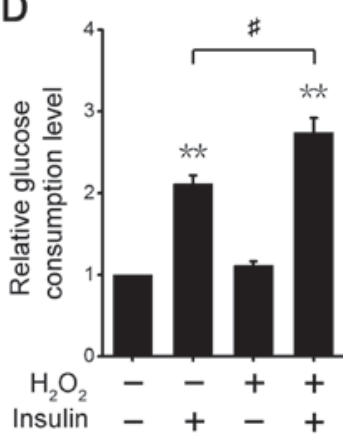

Figure 3. $\mathrm{H}_{2} \mathrm{O}_{2}$ increases insulin-induced GLUT4 translocation and glucose uptake in 3T3-L1 adipocytes. (A) Immunoblot analysis of GLUT4 protein level in the cell membrane of 3T3-L1 adipocytes treated with insulin and/or $\mathrm{H}_{2} \mathrm{O}_{2}$. (B) Quantitative analysis of GLUT4 protein levels in the cell membrane as presented in panel A. (C) 2-NBDG uptake in 3T3-L1 adipocytes under the indicated treatments. (D) Glucose consumption by 3T3-L1 adipocytes in culture medium under the indicated treatments. Data are presented as the mean \pm standard error mean from three independent experiments. ${ }^{*} \mathrm{P}<0.05$ and ${ }^{* *} \mathrm{P}<0.01 \mathrm{vs}$. the control group; ${ }^{\#} \mathrm{P}<0.05$, as indicated. $\mathrm{H}_{2} \mathrm{O}_{2}$, hydrogen peroxide; GLUT4, glucose transporter 4; 2-NBDG, 2-(N-(7-nitrobenz-2-oxa-1,3-diazol-4-yl) amino)-2-deoxyglucose.

Insulin sensitivity is improved in 3T3-L1 adipocytes after transient $\mathrm{H}_{2} \mathrm{O}_{2}$ treatment. PTEN can catalyze the dephosphorylation of $\mathrm{PIP}_{3}$, preventing insulin-induced PI3-K signaling to AKT and downstream of AKT $(12,23)$. However, PTEN, as one of the important targets of ROS, is oxidized at cysteine residues and inactivated (27). Thus, we analyzed PTEN oxidation in 3T3-L1 adipocytes after transient $\mathrm{H}_{2} \mathrm{O}_{2}$ treatment. The cells were lysed in a buffer containing NEM to block free sulfhydryl groups, leading to increasing electrophoretic mobility in the oxidized form of PTEN. As shown in Fig. 2A, transient $\mathrm{H}_{2} \mathrm{O}_{2}$ treatment significantly increased PTEN oxidation in 3T3-L1 adipocytes. The PI3-K/AKT signaling axis regulates insulin-induced metabolic action, including glucose uptake, glycogen synthesis and lipid synthesis (32). Thus, AKT phosphorylation is often used to evaluate the activity of insulin signaling in insulin-response tissues or cells (33). AKT1/2 are activated via phosphorylation of Thr308/309 residues in the catalytic domain and Ser473/474 residues in the hydrophobic motif (34). We further detected AKT phosphorylation levels at Thr308/309 and Ser473/474 residues in 3T3-L1 adipocytes after transient $\mathrm{H}_{2} \mathrm{O}_{2}$ or/and insulin treatment. The result showed that the phosphorylation levels of AKT were significantly enhanced in insulin or/and transient $\mathrm{H}_{2} \mathrm{O}_{2}$-treated 3T3-L1 adipocytes, and that co-treatment with insulin and $\mathrm{H}_{2} \mathrm{O}_{2}$ promoted increased AKT phosphorylation compared with insulin stimulation (Fig. 2B). The statistical analysis was consistent with this result (Fig. 2C). Immunofluorescence analysis also revealed that transient $\mathrm{H}_{2} \mathrm{O}_{2}$ treatment enhanced the activation of insulin-induced AKT in 3T3-L1 adipocytes (Fig. 2D). These data suggested that transient
$\mathrm{H}_{2} \mathrm{O}_{2}$ could enhance AKT activation through the oxidation and inactivation of PTEN, and improve insulin sensitivity in 3T3-L1 adipocytes.

Transient $\mathrm{H}_{2} \mathrm{O}_{2}$ increases insulin-induced GLUT4 translocation and glucose uptake in 3T3-L1 adipocytes. We further analyzed the activation level of AKT-downstream signaling after different treatments in 3T3-L1 adipocytes. GLUT4, which is glucose transport protein that is highly expressed in adipose tissues, shifts from its intracellular location to the cell membrane via insulin-stimulated AKT signaling (35). The cytoplasm and membrane fractions assay showed that insulin increased GLUT4 translocation to the cell membrane, and co-treatment with both insulin and transient $\mathrm{H}_{2} \mathrm{O}_{2}$ also significantly enhanced GLUT4 redistribution in 3T3-L1 adipocytes (Fig. 3A). Consistent with this result, quantitative analysis revealed that the GLUT4 protein level at the cell surface was increased after insulin or/and transient $\mathrm{H}_{2} \mathrm{O}_{2}$ treatment, and the translocation effect with insulin and $\mathrm{H}_{2} \mathrm{O}_{2}$ co-treatment was more marked than with single insulin treatment (Fig. 3B). These results demonstrated that transient ROS increases the effect of insulin on the regulation of GLUT4 protein expression in 3T3-L1 adipocytes.

We next examined changes in glucose uptake ability in 3T3-L1 adipocytes treated with insulin or/and transient $\mathrm{H}_{2} \mathrm{O}_{2}$. The level of intracellular 2-NBDG, a fluorescent glucose, was measured, and the results indicated that the 2-NBDG uptake level was significantly raised in 3T3-L1 adipocytes treated with insulin or/and transient $\mathrm{H}_{2} \mathrm{O}_{2}$ (Fig. 3C). The effect was best after co-stimulation with both insulin and $\mathrm{H}_{2} \mathrm{O}_{2}$ (Fig. 3C). Likewise, we examined the glucose consumption level in the 
A

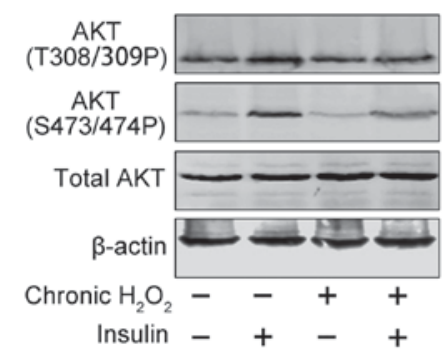

C

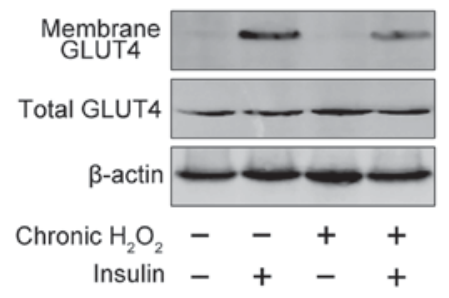

E

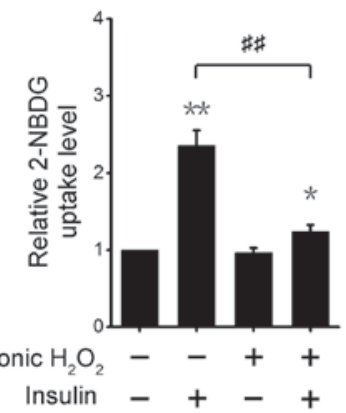

B

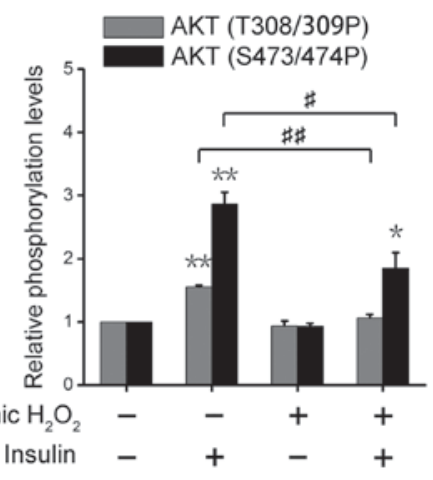

D

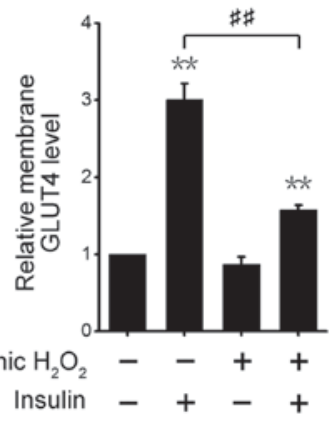

$\mathrm{F}$

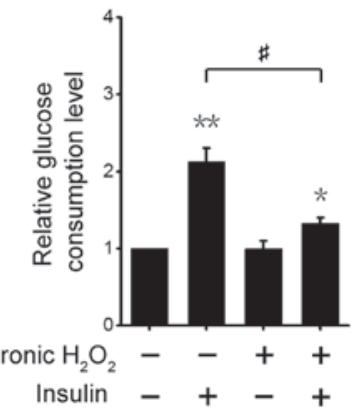

Figure 4. Chronic $\mathrm{H}_{2} \mathrm{O}_{2}$ causes insulin resistance in 3T3-L1 adipocytes. (A) Immunoblot analysis of AKT phosphorylation in glucose oxidase treated or untreated 3T3-L1 adipocytes under the indicated treatment. (B) Quantitative analysis of AKT phosphorylation protein level as presented in panel A. (C) Immunoblot analysis of GLUT4 protein level in the cell membrane of chronic ROS-treated or untreated 3T3-L1 adipocytes with the indicated treatments. (D) Quantitative analysis of GLUT4 protein level in cell membrane as presented in panel C. (E) 2-NBDG uptake in 3T3-L1 adipocytes under the indicated treatments. (F) Glucose consumption in culture medium by 3T3-L1 adipocytes of the different treatments. Data are presented as the mean \pm standard error mean from three independent experiments. ${ }^{*} \mathrm{P}<0.05$ and ${ }^{* *} \mathrm{P}<0.01$ vs. the control group; ${ }^{\#} \mathrm{P}<0.05$ and ${ }^{\# \#} \mathrm{P}<0.01$, as indicated. $\mathrm{H}_{2} \mathrm{O}_{2}$, hydrogen peroxide; GLUT4, glucose transporter 4; AKT, protein kinase B; 2-NBDG, 2-(N-(7-nitrobenz-2-oxa-1,3-diazol-4-yl) amino)-2-deoxyglucose; ROS, reactive oxygen species.

culture medium. The results showed that the three different treatments led to increases in glucose utilization, and transient $\mathrm{H}_{2} \mathrm{O}_{2}$ markedly enhanced insulin-induced glucose consumption in the culture medium (Fig. 3D). These results demonstrated that transient ROS increased the insulin-stimulated glucose uptake level, suggesting that the insulin sensitivity of adipocytes is elevated by transient ROS.

Chronic $\mathrm{H}_{2} \mathrm{O}_{2}$ caused insulin resistance in 3T3-L1 adipocytes. The above data suggested that transient ROS treatment could enhance insulin-regulated metabolic effects (Figs. 2 and 3), thus we further explored metabolic alterations in adipose cells after chronic ROS exposure. As shown in Fig. 4A, incubation with glucose oxidase, which catalyzed the conversion of glucose to glucuronic acid and $\mathrm{H}_{2} \mathrm{O}_{2}$, did not change basic AKT phosphorylation levels; however, the phosphorylation levels of insulin-induced AKT in chronic ROS-treated adipocytes were significantly decreased. Densitometry analysis of phosphorylated AKT also produced the similar results (Fig. 4B). These data suggested that chronic ROS stimulation damaged 3T3-L1 adipocytes in response to insulin. The cytoplasm and membrane fractions assay showed the insulin-stimulated GLUT4 translocation to the cell surface was lower in chronic glucose oxidase-incubated adipocytes than in non-incubated cells (Fig. 4C and D). We further examined the changes in glucose absorption in 3T3-L1 adipocytes under chronic ROS treatment. Insulin promoted 2-NBDG uptake in 3T3-L1 adipocytes incubated with or without glucose oxidase; however, the capability of insulin-stimulated 2-NBDG absorption in chronic ROS-treated adipocytes was strikingly decreased (Fig. 4E). Consistent with this result, glucose consumption was also reduced in insulin-stimulated 3T3-L1 adipocytes incubated with glucose oxidase, compared with enzyme-free incubation (Fig. 4F). These results demonstrated that chronic ROS exposure impaired the insulin sensitivity of 3T3-L1 adipocytes. 


\section{Discussion}

About 40 years ago, many studies reported that insulin can elicit ROS generation in skeletal muscles, adipose tissues, the liver and other target tissues and cells (36). Insulin activates NADPH oxidase, which is a plasma membrane enzyme, leading to $\mathrm{H}_{2} \mathrm{O}_{2}$ production $(10,11,36)$. Our data also showed that insulin increased the intracellular ROS content in 3T3-L1 adipocytes. ROS oxidize and inactivate PTPs, such as PTEN and protein-tyrosine phosphatase-1b (PTP1b), which dephosphorylate insulin-induced phosphorylation to block insulin signaling $(12,18,23)$. However, catalytic activation of PTPs depends on cysteine residues, which are highly susceptible to reversible oxidation and inactivation by ROS (37). A previous study further revealed that an increase in intracellular ROS content enhances insulin regulatory functions, but ROS scavengers attenuate these effects (18). Co-treatment with both $\mathrm{H}_{2} \mathrm{O}_{2}$ and insulin led to stronger metabolic regulation, including AKT activation, GLUT4 translocation and glucose uptake, compared with the single-insulin stimulation. Thus, transient and acute ROS treatment improves insulin sensitivity in adipose cells.

Although ROS have important roles in insulin-regulated metabolic pathways, chronic ROS or oxidative stress could cause activation of intracellular stress-sensitive pathways, resulting in insulin resistance and glucose-lipid metabolic diseases $(38,39)$. ROS activate mitogen-activated protein kinases (MAPKs), such as c-Jun N-terminal kinase (JNK), through oxidation and inactivation of MAPK phosphatases (2). Many studies have also revealed that ROS activate apoptosis signal-regulating kinase 1 (ASK1) by oxidizing thioredoxin (TRX), which binds and inhibits ASK1 activation, leading to activation of the JNK signaling pathway $(40,41)$. In addition, ROS also activate inhibitor of nuclear factor $\kappa-\mathrm{B}$ kinase subunit $\beta$ (IKK $\beta$ ) through $\mathrm{Src}$ and Abl tyrosine kinase signaling pathway (42). ROS-activated JNK and IKK $\beta$ phosphorylate IRS1 at Ser 302, 307 and 612 sites to block IRS1 binding to the IR and promote IRS1 degradation, resulting in attenuation of the delivery of insulin signaling (43). Therefore, chronic ROS treatment can induce and contribute to insulin resistance.

In conclusion, here we clarified that transient $\mathrm{H}_{2} \mathrm{O}_{2}$ enhances insulin sensitivity in 3T3-L1 adipocytes. Mechanistically, $\mathrm{H}_{2} \mathrm{O}_{2}$ treatment may enhance insulin-stimulated AKT activation through the oxidation and inactivation of PTEN compared with single insulin treatment, resulting in increased GLUT4 translocation and glucose uptake. Overall this study provides evidence to better understand the function of ROS in insulin signaling pathways in adipose cells.

\section{Acknowledgements}

Not applicable.

\section{Funding}

The present study was supported by the National Key Research and Development Program of China (grant no. 2017YFA0205200), National Natural Science Foundation of China (grant no. 81571785 and 81771957) and Natural
Science Foundation of Guangdong Province, China (grant nos. 2016A030311055 and 2016A030313770).

\section{Availability of data and materials}

The datasets used and analyzed during the current study are available from the corresponding author on reasonable request.

\section{Authors' contributions}

LLuo, LLu and MM conceived and designed the research. MM, YQ, YL, XH, JX, MZ, WZ and YX performed the experiments. MM processed and analyzed the experimental results. MM and YQ wrote the manuscript. All authors read and approved the manuscript.

\section{Ethics approval and consent to participate}

Not applicable.

\section{Consent for publication}

Not applicable.

\section{Competing interests}

The authors declare that they have no competing interests.

\section{References}

1. Turrens JF: Mitochondrial formation of reactive oxygen species. J Physiol 552: 335-344, 2003.

2. Tiganis T: Reactive oxygen species and insulin resistance: The good, the bad and the ugly. Trends Pharmacol Sci 32: 82-89, 2011.

3. Nishikawa T and Araki E: Impact of mitochondrial ROS production in the pathogenesis of diabetes mellitus and its complications. Antioxid Redox Signal 9: 343-353, 2007.

4. Newsholme P, Haber EP, Hirabara SM, Rebelato EL, Procopio J, Morgan D, Oliveira-Emilio HC, Carpinelli AR and Curi R: Diabetes associated cell stress and dysfunction: Role of mitochondrial and non-mitochondrial ROS production and activity. J Physiol 583: 9-24, 2007.

5. Riemer J, Bulleid N and Herrmann JM: Disulfide formation in the ER and mitochondria: Two solutions to a common process. Science 324: 1284-1287, 2009.

6. Panzhinskiy E, Ren J and Nair S: Protein tyrosine phosphatase $1 \mathrm{~B}$ and insulin resistance: role of endoplasmic reticulum stress/reactive oxygen species/nuclear factor kappa B axis. PLoS One 8: e77228, 2013.

7. Freedman RB, Hirst TR and Tuite MF: Protein disulphide isomerase: Building bridges in protein folding. Trends Biochem Sci 19: 331-336, 1994.

8. Figueira TR, Barros MH, Camargo AA, Castilho RF, Ferreira JC, Kowaltowski AJ, Sluse FE, Souza-Pinto NC and Vercesi AE: Mitochondria as a source of reactive oxygen and nitrogen species: From molecular mechanisms to human health. Antioxid Redox Sign 18: 2029-2074, 2013.

9. Bedard K and Krause KH: The NOX family of ROS-generating NADPH oxidases: Physiology and pathophysiology. Physiol Rev 87: 245-313, 2007.

10. Mukherjee SP, Lane RH and Lynn WS: Endogenous hydrogen peroxide and peroxidative metabolism in adipocytes in response to insulin and sulfhydryl reagents. Biochem Pharmacol 27: 2589-2594, 1978.

11. May JM and de Haën C: Insulin-stimulated intracellular hydrogen peroxide production in rat epididymal fat cells. J Biol Chem 254: 2214-2220, 1979. 
12. Seo JH, Ahn Y, Lee SR, Yeol Yeo C and Chung Hur K: The major target of the endogenously generated reactive oxygen species in response to insulin stimulation is phosphatase and tensin homolog and not phosphoinositide-3 kinase (PI-3 kinase) in the PI-3 kinase/Akt pathway. Mol Biol Cell 16: 348-357, 2005

13. Gericke A, Munson M and Ross AH: Regulation of the PTEN phosphatase. Gene 374: 1-9, 2006.

14. Ross AH and Gericke A: Phosphorylation keeps PTEN phosphatase closed for business. Proc Natl Acad Sci USA 106: 1297-1298, 2009.

15. Taniguchi CM, Emanuelli B and Kahn CR: Critical nodes in signalling pathways: Insights into insulin action. Nat Rev Mol Cell Biol 7: 85-96, 2006.

16. Cantley LC: The phosphoinositide 3-kinase pathway. Science 296: 1655-1657, 2002.

17. Cheng CK, Fan QW and Weiss WA: PI3K signaling in glioma-animal models and therapeutic challenges. Brain pathol 19: 112-120, 2009.

18. Loh K, Deng H, Fukushima A, Cai X, Boivin B, Galic S, Bruce C, Shields BJ, Skiba B, Ooms LM, et al: Reactive oxygen species enhance insulin sensitivity. Cell Metab 10: 260-272, 2009.

19. Whelan SA, Dias WB, Thiruneelakantapillai L, Lane MD and Hart GW: Regulation of insulin receptor substrate 1 (IRS-1)/AKT kinase-mediated insulin signaling by O-Linked beta- $\mathrm{N}$-acetylglucosamine in $3 \mathrm{~T} 3-\mathrm{L} 1$ adipocytes. J Biol Chem 285: 5204-5211, 2010.

20. Cong LN, Chen H, Li Y, Zhou L, McGibbon MA, Taylor SI and Quon MJ: Physiological role of Akt in insulin-stimulated translocation of GLUT4 in transfected rat adipose cells. Mol Endocrinol 11: 1881-1890, 1997.

21. Favaretto F, Milan G, Collin GB, Marshall JD, Stasi F, Maffei P, Vettor R and Naggert JK: GLUT4 defects in adipose tissue are early signs of metabolic alterations in Alms1GT/GT, a mouse model for obesity and insulin resistance. PLoS One 9: e109540, 2014.

22. Chen YH, Heneidi S, Lee JM, Layman LC, Stepp DW, Gamboa GM, Chen BS, Chazenbalk G and Azziz R: miRNA-93 inhibits GLUT4 and is overexpressed in adipose tissue of polycystic ovary syndrome patients and women with insulin resistance. Diabetes 62: 2278-2286, 2013.

23. Nakashima N, Sharma PM, Imamura T, Bookstein R and Olefsky JM: The tumor suppressor PTEN negatively regulates insulin signaling in 3T3-L1 adipocytes. J Biol Chem 275 12889-12895, 2000

24. Mehlem A, Hagberg CE, Muhl L, Eriksson U and Falkevall A: Imaging of neutral lipids by oil red $\mathrm{O}$ for analyzing the metabolic status in health and disease. Nat Protoc 8: 1149-1154, 2013.

25. Potashnik R, Bloch-Damti A, Bashan N and Rudich A: IRS1 degradation and increased serine phosphorylation cannot predict the degree of metabolic insulin resistance induced by oxidative stress. Diabetologia 46: 639-648, 2003.

26. Yang $\mathrm{P}$, Zhao Y, Zhao L, Yuan J, Chen Y, Varghese Z, Moorhead JF, Chen Y and Ruan XZ: Paradoxical effect of rapamycin on inflammatory stress-induced insulin resistance in vitro and in vivo. Sci Rep 5: 14959, 2015

27. Lee SR, Yang KS, Kwon J, Lee C, Jeong W and Rhee SG: Reversible inactivation of the tumor suppressor PTEN by $\mathrm{H} 2 \mathrm{O} 2$. J Biol Chem 277: 20336-20342, 2002.

28. Jiang $X$, Huang $L$ and Xing D: Photoactivation of Dok1/ERK/PPAR $\gamma$ signaling axis inhibits excessive lipolysis in insulin-resistant adipocytes. Cell Signal 27: 1265-1275, 2015.
29. Bernlohr DA, Bolanowski MA, Kelly TJ Jr and Lane MD: Evidence for an increase in transcription of specific mRNAs during differentiation of 3T3-L1 preadipocytes. J Biol Chem 260: 5563-5567, 1985 .

30. Turpin SM, Nicholls HT, Willmes DM, Mourier A, Brodesser S, Wunderlich CM, Mauer J, Xu E, Hammerschmidt P, Brönneke HS, et al: Obesity-induced CerS6-dependent C16:0 ceramide production promotes weight gain and glucose intolerance. Cell Metab 20: 678-686, 2014.

31. Quan YY, Qin GQ, Huang H, Liu YH, Wang XP and Chen TS Dominant roles of Fenton reaction in sodium nitroprusside-induced chondrocyte apoptosis. Free Radic Biol Med 94: 135-144, 2016.

32. Whiteman EL, Cho $\mathrm{H}$ and Birnbaum MJ: Role of Akt/protein kinase B in metabolism. Trends Endocrinol Metab 13: 444-451, 2002.

33. Gonzalez E, Flier E, Molle D, Accili D and McGraw TE: Hyperinsulinemia leads to uncoupled insulin regulation of the GLUT4 glucose transporter and the FoxO1 transcription factor. Proc Natl Acad Sci USA 108: 10162-10167, 2011.

34. Koseoglu S, Lu Z, Kumar C, Kirschmeier P and Zou J: AKT1, AKT2 and AKT3-dependent cell survival is cell line-specific and knockdown of all three isoforms selectively induces apoptosis in 20 human tumor cell lines. Cancer Biol Ther 6: 755-762, 2007.

35. Bryant NJ, Govers R and James DE: Regulated transport of the glucose transporter GLUT4. Nat Rev Mol Cell Biol 3: 267-277, 2002.

36. Goldstein BJ, Mahadev K, Wu X, Zhu L and Motoshima H: Role of insulin-induced reactive oxygen species in the insulin signaling pathway. Antioxid Redox Signal 7: 1021-1031, 2005.

37. Tonks NK: Protein tyrosine phosphatases: From genes, to function, to disease. Nat Rev Mol Cell Biol 7: 833-846, 2006.

38. Bloch-Damti A and Bashan N: Proposed mechanisms for the induction of insulin resistance by oxidative stress. Antioxid Redox Signal 7: 1553-1567, 2005.

39. Ding H, Heng B, He W, Shi L, Lai C, Xiao L, Ren H, Mo S and $\mathrm{Su} \mathrm{Z}$ : Chronic reactive oxygen species exposure inhibits glucose uptake and causes insulin resistance in $\mathrm{C} 2 \mathrm{C} 12$ myotubes. Biochem Biophys Res Commun 478: 798-803, 2016

40. Matsukawa J, Matsuzawa A, Takeda K and Ichijo H: The ASK1-MAP kinase cascades in mammalian stress response. J Biochem 136: 261-265, 2004.

41. Tobiume K, Matsuzawa A, Takahashi T, Nishitoh H, Morita K, Takeda K, Minowa O, Miyazono K, Noda T and Ichijo H: ASK1 is required for sustained activations of $\mathrm{JNK} / \mathrm{p} 38$ MAP kinases and apoptosis. EMBO Rep 2: 222-228, 2001.

42. Storz $\mathrm{P}$ and Toker A: Protein kinase D mediates a stress-induced NF-kappaB activation and survival pathway. EMBO J 22: 109-120, 2003

43. Gual P, Le Marchand-Brustel Y and Tanti JF: Positive and negative regulation of insulin signaling through IRS-1 phosphorylation. Biochimie 87: 99-109, 2005.

(i) $\ominus$ This work is licensed under a Creative Commons Attribution-NonCommercial-NoDerivatives 4.0 International (CC BY-NC-ND 4.0) License. 